\title{
Spin-Polarized Current in Coulomb Blockade and Kondo Regime
}

\author{
P. OGRODNIK* AND R. ŚwirkOWICZ \\ Faculty of Physics, Warsaw University of Technology \\ Koszykowa 75, 00-662 Warsaw, Poland
}

\begin{abstract}
Spin-polarized electron transport through a single-level quantum dot attached to ferromagnetic and non-magnetic leads is studied with the use of the non-equilibrium Green function technique. Current polarization is investigated in dependence on parameters which characterize the device: the lead's polarization, a position of the dot energy level compared to the Fermi level in electrodes, and the Zeeman splitting of the dot level in an external magnetic field applied to the dot. Calculations were performed in two transport regimes: the Coulomb blockade regime with correlation effects treated within the Hartree-Fock approximation and the Kondo regime with a more rigorous approach to many-body effects at low temperatures.
\end{abstract}

PACS numbers: 73.23.-b, 73.21.La, 85.75.--d

\section{Introduction}

In recent years, much of interest has been focused on spintronics, the new field of research, in which an additional degree of freedom, electron spin, plays an important role. Possibilities of generating and operating highly spin-polarized currents are fundamental features of spintronic devices. Spin polarization of the current is usually defined as $P=\left(I_{\uparrow}-I_{\downarrow}\right) /\left(I_{\uparrow}+I_{\downarrow}\right)$ with $I_{\sigma}$ denoting the current flowing in a channel corresponding to spin $\sigma=\uparrow(\downarrow)$. Recently, a variety of spin filters, systems acting as generators of spin-polarized current, has been proposed. Among them, one can find planar tunnel junctions with ferromagnetic barriers, where effective exchange field leads to a splitting of the band into spin-majority and spin-minority sub-bands which results in a spin-dependent transmission [1]. Furthermore, spin filters based on quantum interferences have been proposed, in which electrons with the one spin interfere constructively whereas with the opposite spin interfere destructively, so a flow of carriers with one spin direction is permitted in the system [2]. Another interesting proposal is a spin filter based on an idea of application of the well-known Stern-Gerlach effect in quantum wires [3]. Moreover,

${ }^{*}$ corresponding author; e-mail: piotrogr@if.pw.edu.pl 
many semiconductors with a sufficiently large spin-orbital interaction can work as components of spin filters and there are known quantum tunnelling structures based on such materials through which spin-polarized current can flow [4]. There are also many examples of using quantum dots (QDs) as small and effective spin filters. In this work we present an idea of application as a spin filter of a single-level QD attached to one ferromagnetic and to one non-magnetic electrodes.

\section{Model}

A QD is described by the Hamiltonian

$$
H_{\mathrm{D}}=\sum_{\sigma} E_{\sigma} d_{\sigma}^{+} d_{\sigma}+U d_{\uparrow}^{+} d_{\uparrow} d_{\downarrow}^{+} d_{\downarrow}
$$

where $E_{\sigma}$ represents the spin-dependent energy level in the dot and $U$ is the parameter describing electron correlations. The dot is connected via tunnelling barriers to ferromagnetic (or non-magnetic) electrodes treated as reservoirs of free electrons. Tunnelling processes between QD and electrodes are described by the Hamiltonian

$$
H_{\mathrm{T}}=\sum_{k \beta \sigma}\left(T_{k \beta}^{\sigma} a_{k \beta \sigma}^{+} d_{\sigma}+\text { h.c. }\right)
$$

where $a_{k \beta \sigma}^{+}$represents a creation operator of an electron with energy $\varepsilon_{k \beta \sigma}$ in the electrode $\beta$ ( $\beta=\mathrm{L}$ or $\beta=\mathrm{R})$ and $T_{k \beta}^{\sigma}$ are elements of tunnelling matrix which are related to tunnelling rates

$$
\Gamma_{\sigma}^{\beta}=2 \pi \sum_{k}\left|T_{k \beta}^{\sigma}\right|^{2} \delta\left(\varepsilon-\varepsilon_{k \beta \sigma}\right) .
$$

In the following it is assumed: $\Gamma_{\sigma}^{\beta}=\Gamma_{0}\left(1+\hat{\sigma} p_{\beta}\right)$, where $\hat{\sigma}= \pm 1, p_{\beta}$ is a factor describing spin polarization in the lead $\beta$ and $\Gamma_{0}$ is constant within electron band of width $D$ and zero otherwise.

Electric current in a channel corresponding to spin $\sigma$ is calculated according to the formula derived by Meir et al. [5]:

$$
\begin{aligned}
I^{\sigma}= & \mathrm{i} \frac{e}{2 \hbar} \int \frac{\mathrm{d} \varepsilon}{2 \pi}\left\{\left(\Gamma_{\sigma}^{\mathrm{L}}-\Gamma_{\sigma}^{\mathrm{R}}\right) G_{\sigma}^{<}(\varepsilon)\right. \\
& \left.+\left[\Gamma_{\sigma}^{\mathrm{L}} f_{\mathrm{L}}(\varepsilon)-\Gamma_{\sigma}^{\mathrm{R}} f_{\mathrm{R}}(\varepsilon)\right]\left[G_{\sigma}^{\mathrm{r}}(\varepsilon)-G_{\sigma}^{\mathrm{a}}(\varepsilon)\right]\right\} .
\end{aligned}
$$

$f_{\beta}$ represents here the Fermi-Dirac distribution function and $G_{\sigma \sigma^{\prime}}^{i}=\left\langle\left\langle d_{\sigma}, d_{\sigma^{\prime}}^{+}\right\rangle\right\rangle_{\varepsilon}^{i}$ denotes the lesser, retarded, and advanced Green function for $i=<, \mathrm{r}$, a, respectively. $G_{\sigma}^{<}$is found from the Keldysh formula $G_{\sigma}^{<}=G_{\sigma}^{\mathrm{r}} \Sigma_{\sigma}^{<} G_{\sigma}^{\mathrm{a}}$ with the self-energy given by the standard expression $\Sigma_{\sigma}^{<}=\mathrm{i}\left(\Gamma_{\sigma}^{\mathrm{L}} f_{\mathrm{L}}+\Gamma_{\sigma}^{\mathrm{R}} f_{\mathrm{R}}\right)$, whereas $G_{\sigma}^{\mathrm{r}}, G_{\sigma}^{\mathrm{a}}$ are determined from the equation of motion. Two different decoupling schemes are used, namely, the standard Hartree-Fock approximation (HFA), which allows one to describe transport in the Coulomb blockade regime at relatively high temperatures as well as the procedure appropriate in the Kondo regime in a low temperature region. The Green function calculated within HFA is given by the 
formula

$$
G_{\sigma}^{\mathrm{r}(\mathrm{HFA})}=\frac{1-n_{-\sigma}}{\varepsilon-E_{\sigma}-\Sigma_{0 \sigma}^{\mathrm{r}}}+\frac{n_{-\sigma}}{\varepsilon-E_{\sigma}-U-\Sigma_{0 \sigma}^{\mathrm{r}}}
$$

with $\Sigma_{0 \sigma}^{\mathrm{r}}=-\frac{\mathrm{i}}{2}\left(\Gamma_{\sigma}^{\mathrm{L}}+\Gamma_{\sigma}^{\mathrm{R}}\right)$ and occupation numbers $n_{\sigma}=-\mathrm{i} \int \frac{\mathrm{d} \varepsilon}{2 \pi} G_{\sigma}^{<}$. In the Kondo regime instead of $\Sigma_{0 \sigma}^{\mathrm{r}}$ a much more complicated expression is obtained for the self-energy. However, considering the limit $U \rightarrow \infty$ one can write the Green function in the simple form

$$
G_{\sigma}^{\mathrm{r}(\mathrm{K})}=\frac{1-n_{-\sigma}}{\varepsilon-E_{\sigma}-\left(\Sigma_{0 \sigma}^{\mathrm{r}}+\Sigma_{1 \sigma}^{\mathrm{r}}\right)},
$$

where

$$
\Sigma_{1 \sigma}^{\mathrm{r}}=\sum_{\beta} \int \frac{\mathrm{d} E}{2 \pi} \frac{\Gamma_{-\sigma}^{\beta} f_{\beta}(E)}{\varepsilon-E_{\sigma}+E_{-\sigma}-E+\mathrm{i} \hbar / \tau_{-\sigma}}
$$

and $\tau_{\sigma}$ is the spin-dependent relaxation time [5].

\section{Results and discussion}

Transport properties have been studied for a QD attached to a ferromagnetic electrode acting as a drain for the positive bias $(e V>0)$ and to a non-magnetic one, being a source for positive bias. Calculations have been performed in two limits: in the Coulomb blockade regime with $k T=\Gamma_{0}=0.025, U=1$ and in the Kondo regime with $k T=0.001, \Gamma_{0}=0.1$ (measured in units $\gamma=D / 50$ ).

\subsection{Coulomb blockade regime}

First, we consider the case with an external magnetic field applied to the dot which leads to the splitting of the dot level in the way: $E_{\sigma}=E_{0}+\frac{1}{2} \hat{\sigma} \Delta$. Results, as depicted in Fig. 1, show that highly polarized current can be observed in a region of relatively small source-drain voltages. Polarization characteristics $P(e V)$ are strongly asymmetric with respect to the bias reversal and depend considerably on the level position $E_{0}$ as well as on the splitting $\Delta$ in the magnetic field. If the dot is empty in the equilibrium state, the effect of bipolarity can be observed as depicted in Figs. 1a and b. With voltage increasing, the first level $E_{\downarrow}$, weakly coupled to the ferromagnetic electrode, enters the bias window and a relatively small but highly polarized current can flow through the system. For higher values of voltage the sign of the polarization changes as the transport occurs mainly in the $\sigma=\uparrow$ channel, strongly coupled to the ferromagnetic electrode. However, currents related to positive and negative polarizations are considerably different. Especially, the highly polarized current flowing under a low bias voltage is very small (the inset in Fig. 1a). It can be increased by reducing the electrode's polarization $p$, but the bipolarity effect is then less pronounced (Fig. 1b).

Polarization as a function of the bias $P(\mathrm{eV})$ strongly depends on the ratio $\Delta / U$ as depicted in Fig. 1c for the case of fully occupied dot. For $\Delta / U>1$ (dashed line), apart from the Coulomb blockade region, the current polarization 


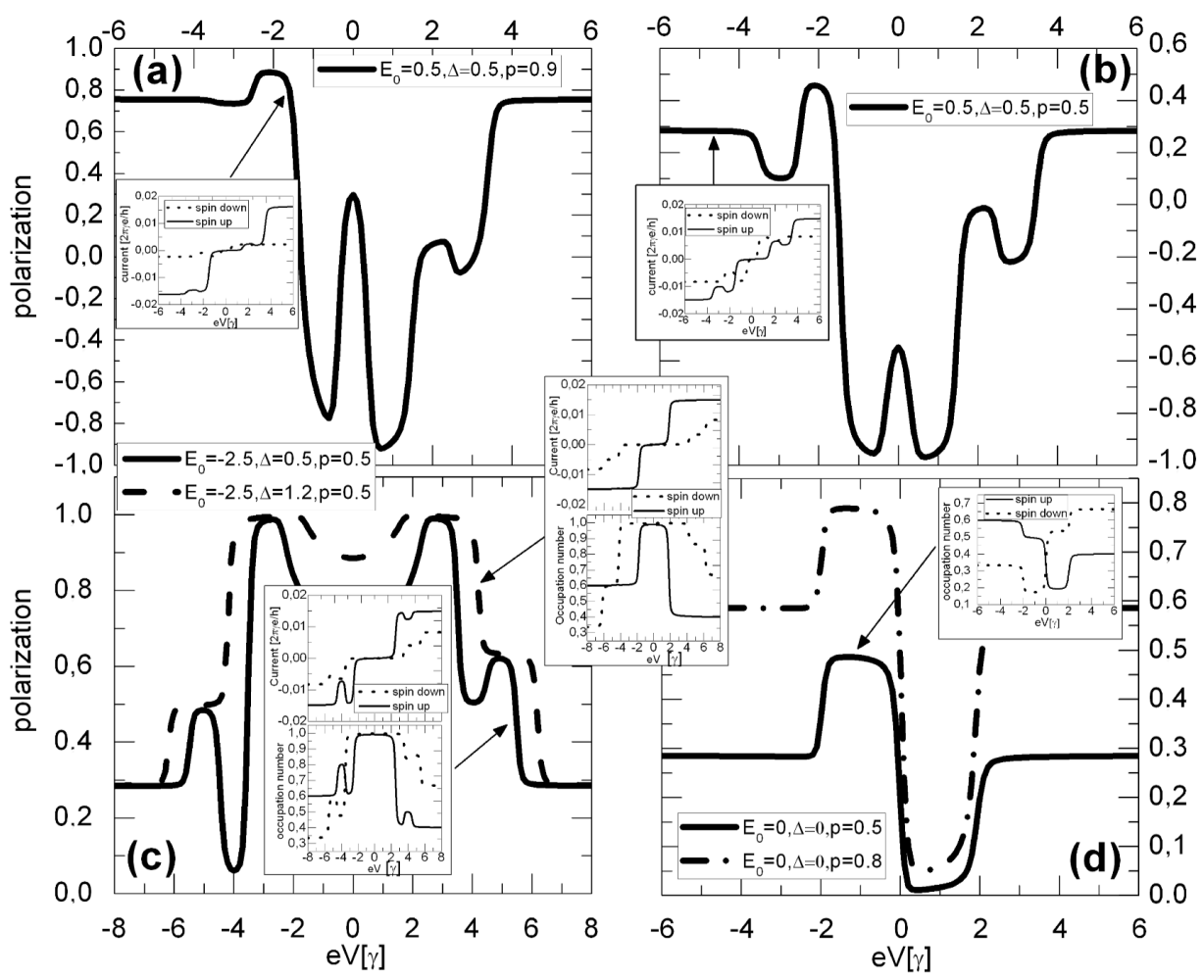

Fig. 1. Polarization vs. source-drain voltage for $\Delta \neq 0(\mathrm{a}-\mathrm{c})$ and for $\Delta=0$ (d) and presented level positions $E_{0}$. Current and occupation numbers vs. bias are given in insets.

decreases monotonically with bias voltage increasing. A different behaviour can be observed for $\Delta / U<1$ (solid line). If $|e V|$ increases, the current polarization is considerably suppressed, then it increases and drops again. These changes are much more pronounced for negative bias $(e V<0)$. As the following relations between energy levels are fulfilled $E_{0 \downarrow}<E_{0 \uparrow}<E_{0 \downarrow}+U<E_{0 \uparrow}+U$, the transport at relatively small voltages occurs mainly in the spin $\uparrow$-channel through the level $E_{0 \uparrow}+U$, which lies in the vicinity of the Fermi energy in the leads. When the level corresponding to spin $\downarrow$ with energy $E_{0 \downarrow}+U$ approaches the bias window and becomes active in the transport, electrons with spin $\uparrow$ start to accumulate on the dot (the left inset in Fig. 1c). Then, the current in the spin $\uparrow$-channel is blocked and lowers considerably, whereas it increases in the channel with spin $\downarrow$. As a result the polarization is substantially suppressed. $P$ increases again at higher voltages when the level $E_{0 \uparrow}$ starts to be active in the transport and the occupation number $n_{\uparrow}$ lowers and becomes constant. Such changes do not occur for $\Delta / U>1$ (the right inset in Fig. 1c). 
Next, we consider the case $\Delta=0$ when the dot can act as a spin diode (Fig. 1d). In the region of small positive values of $e V$ the current polarization is close to 0 , whereas for negative bias spin-polarized current can flow through the system. Such a diode behaviour results from the asymmetry in the coupling strengths to magnetic and non-magnetic electrodes and it is related to specific changes in occupation numbers for both spin directions. According to the inset in Fig. 1d it can be seen that for the positive bias $n_{\downarrow}$ increases considerably. As the transmission through the dot for electrons with spin $\uparrow$, in a low energy region, is weighted by $1-n_{\downarrow}$ (see the expression for $G_{\sigma}^{\mathrm{r}(\mathrm{HFA})}$ ), the current in this spin channel is strongly suppressed. Therefore, despite different couplings to the electrodes it is possible to obtain currents of comparable magnitudes in both channels. For $\mathrm{eV}<0$ the mean number of electrons $n_{\downarrow}$ is getting low, thus a transmission is enhanced for electrons with spin $\uparrow$ and spin polarization of the current is considerable. Magnitude of the current polarization increases with electrode's polarization. The results obtained here are well consistent with those presented by Souza et al. with the use of a different approach [6].

\subsection{Kondo regime}

A non-symmetrical system with the left electrode non-magnetic and the right one ferromagnetic with polarization $p=0.5$ is considered. Due to the coupling to the ferromagnetic electrode the exchange field is generated which acts on the dot and leads to the spin splitting of the dot level similarly as the external magnetic field does [7]. Differential conductance $G_{\text {diff }}^{\sigma}=\mathrm{d} I^{\sigma} / \mathrm{d} V$, as depicted in the inset
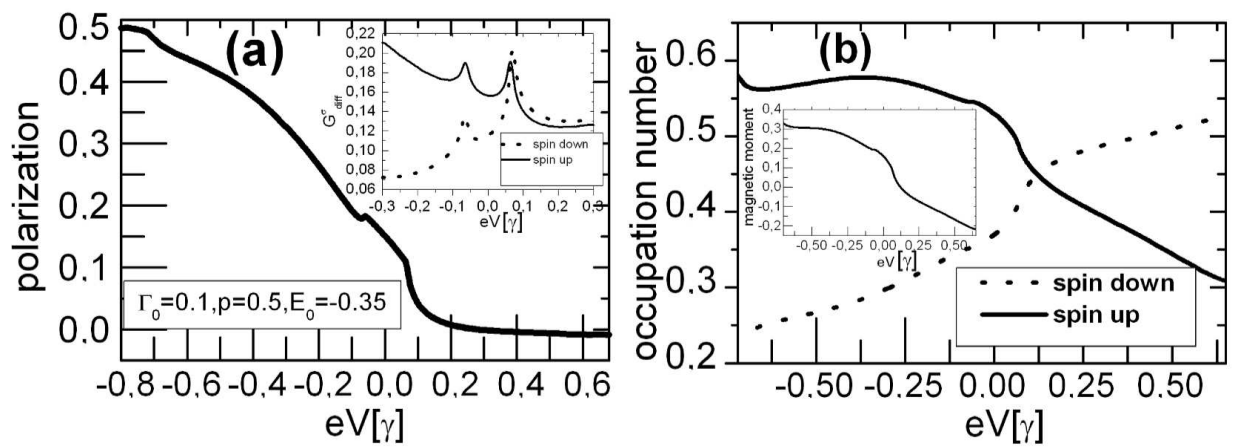

Fig. 2. (a) Current polarization (differential conductance in the inset) and (b) occupation numbers (magnetic moment in the inset) vs. source-drain voltage in the Kondo regime.

in Fig. 2a, reveals the Kondo anomaly in both spin channels, but each zero-bias resonance is split into two components which results from the presence of the

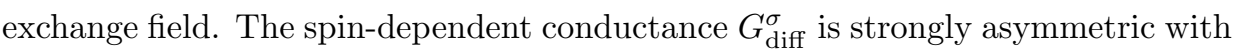
respect to the bias reversal, especially for $\sigma=\downarrow$. For negative values of $\mathrm{eV}$ transport of electrons with spin $\uparrow$ corresponding to the majority spin in the ferromagnetic 
electrode, acting as a source, is considerably enhanced and $G_{\text {diff }}^{\uparrow}$ is substantially higher than $G_{\text {diff. }}^{\downarrow}$ However, for $e V>0$ (positive bias) the channel with minority spins $\downarrow$ becomes very active which results in a strong increase in the appropriate conductance which shows a high and well-pronounced peak. In the region of higher voltages the conductance in both channels is similar and dependence on spin is strongly suppressed. Such effects result in a strong asymmetry in the current polarization. As depicted in Fig. 2a $P$ is considerably higher for negative values of $e V$, but it decreases with an increase in energy and is nearly equal to zero for positive bias. A slight increase in $P$ can be seen in a vicinity of the zero bias voltage, where the Kondo effect manifests its presence. However, it practically does not influence the diode-like behaviour of the current polarization.

Occupation numbers in the dot for both spin polarizations are presented in Fig. 2b. For negative bias the mean number of electrons with spin $\uparrow-n_{\uparrow}$ increases with $|e V|$ increasing, whereas $n_{\downarrow}$ decreases which leads to increase in the magnetic moment $m=n_{\uparrow}-n_{\downarrow}$ accumulated on the dot. For positive bias the dependence is more complex. In a low bias region $(e V<0.1)$ the moment $m$ decreases to zero as depicted in the inset to Fig. 2b, then, changes the sign and its magnitude increases. Such a behaviour originates from the strong asymmetry in the tunnelling rates to both electrodes and considerably influences spin-dependent transport.

Summarizing the results we state that the spin-diode behaviour can be observed for asymmetrical system with one ferromagnetic electrode in the Coulomb blockade and the Kondo regimes.

\section{References}

[1] T.F. Santos, J.S. Moodera, Phys. Rev. B 69, 241203 (2004).

[2] A. Folk, R.M. Potok, C.M. Marcus, V. Umansky, Science 299, 679 (2003).

[3] J. Wróbel, T. Dietl, A. Łusakowski, G. Grabecki, K. Fronc, R. Hey, K.H. Ploog, H. Shtrikman, Phys. Rev. Lett. 93, 246601 (2004).

[4] Leo Yu, H.C. Huang, O. Voskoboynikov, Superlatt. Microstruct. 34, 547 (2003).

[5] Y. Meir, N.S. Wingreen, P.A. Lee, Phys. Rev. Lett. 70, 2601 (1993).

[6] F.M. Souza, J.C. Egues, A.P. Jauho, Phys. Rev. B 75, 165303 (2007).

[7] R. Świrkowicz, M. Wilczyński, J. Barnaś, J. Phys., Condens. Matter 18, 2291 (2006). 